\section{Latente Tuberkulose in Niedriginzidenz-Region durch Einwanderung bestimmt}

Dale KD et al. Estimating the prevalence of latent tuberculosis in a low-incidence setting: Australia. Eur Respir J 2018; 52: doi:10.1183/ 13993003.01218-2018

In Niedriginzidenz-Ländern ist Migration ein wesentlicher Treiber für den Anstieg der Tuberkulose(TB)-Infektionen - meist in Form einer Reaktivierung von in den Herkunftsländern erworbenen latenten Tuberkuloseerkrankungen (LTBI). Deshalb ist die Abschätzung des LTBI-Risikos in unterschiedlichen Migrantenpopulationen ein wichtiger Faktor für die Planung von Maßnahmen und Ressourcen im Gesundheitswesen von Niedriginzidenz-Ländern.

Eine australische Gruppe von Wissenschaftlern um Katie D. Dale vom Tuberkuloseprogramm in Melbourne (Viktoria, Australien) nutzten die australischen Zensusdaten von 2006, 2011 und 2016, um das jährliche Risiko von TB-Infektionen der Populationen anhand des Geburtslandes, des Jahres der Ankunft in Australien und des Alters abzuschätzen. Dabei legten sie TB-Prävalenz-Schätzungen aus den Herkunftsländern weltweit zugrunde.

\section{Ergebnisse}

Der Anteil von Personen mit LTBI in der in Australien geborenen Kohorte war im Zeitraum von 2006 bis 2016 mit 0,4\% gleich geblieben. Der Anteil der Einwohner mit Migrationshintergrund und LTBI war sehr viel höher, hatte sich aber ebenfalls über die Zeit kaum verändert (18,0\% in $2006,17,1 \%$ in 2016). Allerdings war der Anteil der Migranten an der Bevölkerung von 23,8\% in 2006 auf 28,3 \% in 2016 angestiegen. Damit ergab sich auch ein Anstieg der absoluten Zah von LTBI-Betroffenen in Australien von
838000 in 2006 auf 1084000 in 2016. Auch der Anteil der Einwohner Australiens mit LTBI stieg dadurch von 4,6\% (Interquartilrange [IQR] 4,2-5,2) in 2006 auf 5,1\% (IQR 4,7-5,5\%) in 2016 an.

Von allen Einwohnern mit LTBI in 2016 waren $93,2 \%$ in Überseegebieten geboren, am häufigsten in den TB-Hochinzidenz-Ländern Indien, China und den Philippinen. Die nach der Schätzung in jüngerer Zeit leicht sinkenden Zahlen der LTBI-Prävalenz unter Migranten reflektieren die abnehmende Inzidenz in einigen für Australien wichtigen Herkunftsländern wie Indien und China. 21,6\% der LTBI-Träger waren jünger als 35 Jahre und 34,4\% waren seit 2007 nach Australien eingewandert.

Bislang standen bei Screening-Maßnahmen vor allem Flüchtlinge im Fokus der Bemühungen. Diese machen in Australien allerdings nur 2-3\% alle Migranten aus. Das Augenmerk müsste sich nach Meinung der Autoren deshalb viel stärker auch auf andere Populationen aus Hochinzidenz-Ländern richten.

FAZIT

Australien hat eine insgesamt relativ niedrige LTBI-Prävalenz. Allerdings ist die LTBI-Rate bei Migranten aus Hochprävalenz-Ländern hoch und die Reaktivierungsraten könnten insbesondere bei denjenigen, die erst vor kurzem eingewandert sind, relativ hoch sein. Dies spricht für gezielte Screening-Maßnahmen in bestimmten Migrantenpopulationen aus Hochprävalenz-Regionen, um dem Ziel der weltweiten Elimination der TB näher zu kommen.

Friederike Klein, München 